\title{
OPEN ACYCLIC 3-MANIFOLDS, A LOOP THEOREM AND THE POINCARE CONJECTURE ${ }^{1}$
}

\author{
BY T. L. THICKSTUN
}

In [3] the 3-dimensional Poincaré conjecture (hereafter denoted P. C.) was reduced to certain questions about open, irreducible, acyclic 3-manifolds. That reduction is strengthened here.

Theorem 1. P. C. iff every open, irreducible, acyclic 3-manifold, which is the degree one proper image of an open 3-manifold embeddable in $S^{3}$, is also embeddable in $S^{3}$.

Definitions. A 3-manifold, $U^{3}$, is irreducible iff every 2 -sphere, P.L. embedded in $U^{3}$, bounds a 3-ball in $U^{3}$. A map is proper iff the preimage of any compactum is a compactum.

Let $f: V^{3} \rightarrow U^{3}$ be a proper map of open, orientable 3-manifolds (not necessarily connected) and $M^{3}$ a compact 3-submanifold of $V^{3}$ such that $f$ is transverse to $M^{3}$. Denote $N^{3}=f^{-1}\left(M^{3}\right)$. Then $\left.f\right|_{N^{3}}:\left(N^{3}, \partial N^{3}\right) \rightarrow\left(M^{3}, \partial M^{3}\right)$. Furthermore if $U^{3}$ and $V^{3}$ are oriented their orientations induce orientations on $M^{3}$ and $N^{3}$. Corresponding to these orientations we have elements $\alpha_{M} \in$ $H_{3}\left(M^{3}, \partial M^{3}\right)$ and $\beta_{N} \in H_{3}\left(N^{3}, \partial N^{3}\right) . f$ is degree one iff there exist orientations of $U^{3}$ and $V^{3}$ such that for every such $M$ and $N$ as above $f_{*}\left(\beta_{N}\right)=\alpha_{M}$. Given such an $f$ we say $U^{3}$ is a proper degree one image of $V^{3}$. A space is acyclic iff its first homology group with $\mathbf{Z}$ coefficients is trivial. A noncompact 3-manifold, $P^{3}$, is acyclic at $\infty$ iff given any compact subset, $X$, of $P^{3}$ there exists a compact subset, $Y$, of $P^{3}$ such that $X \subset Y$ and $i_{*}: H_{1}\left(P^{3}-Y\right) \rightarrow H_{1}\left(P^{3}-X\right)$ is trivial (where $i: P^{3}-Y \rightarrow P^{3}-X$ is inclusion).

Note. Acyclic implies acyclic at $\infty$.

A virtual disk is a space homeomorphic to $D^{2}-X$ where $X$ is a (generally nonpolyhedral) compact subspace of $\stackrel{\circ}{D}^{2}$ (where $D^{2}$ is the 2-disk). A proper map $f: \breve{D} \rightarrow U^{3}$, of a virtual disk $\breve{D}$, is a virtual disk in $U^{3}$ (referred to as a virtual disk if the range is already specified). If $f$ is an embedding then it is an embedded virtual disk.

Notation. Given $\alpha: S^{1} \rightarrow X,[\alpha]$ will denote the conjugacy class of $\pi_{1}(X)$ determined by $\alpha$.

Received by the editors August 11, 1980.

AMS (MOS) subject classifications (1970). Primary 55A40; Secondary 57 A40.

1 This research was supported by SRC grant GR/A/5561.5. 
The following lemma (the loop theorem of the title) is an analogue for virtual disks, of the classical loop theorem of Stallings.

LEMmA. Let $P^{3}$ be a noncompact 3-manifold with boundary, which is acyclic at $\infty$. Let $\partial_{0} P^{3}$ be a connected component of $\partial P^{3}$ and $A$ a normal subgroup of $\pi_{1}\left(\partial_{0} P^{3}\right)$. Let $f:(\check{D}, \partial \check{D}) \rightarrow\left(P^{3}, \partial_{0} P^{3}\right)$ be a virtual disk such that $\left[\left.f\right|_{\partial \check{D}}\right] \not \subset A$. Then there exists an embedded virtual disk $h:(\check{F}, \partial \check{F}) \rightarrow\left(P^{3}, \partial_{0} P^{3}\right)$ such that $\left[\left.h\right|_{\partial \check{F}}\right] \not \subset A$.

INDICATION OF PROOF. We merely list some of the major ingredients. The tower construction of Papakyriakopoulos is used but rather differently from the way it is used classically. The crucial difficulty involves performing Dehn cuts. Devices are introduced in order to avoid the use of Dehn cuts altogether. Repeated use is made of the main idea of the proof of the "plane theorem" of [2] and Dehn's lemma is used infinitely many times.

OUTLINE OF THE PROOF OF THEOREM 1. If: Given a homotopy 3-sphere $M^{3}$, let $K$ be a Heegaard splitting of $M^{3}$ (by definition, a solid handlebody in $M^{3}$ whose closed complement is also a solid handlebody). By [4] there exists $U^{3}$, an open, irreducible, acyclic 3-manifold, such that $K \subset U^{3} \subset M^{3}$. Let $f: S^{3} \rightarrow$ $M^{3}$ be a degree one map transverse to $U^{3}$ (i.e. transverse to each element of an infinite sequence of compact 3-submanifolds of $U^{3}$ which exhausts $U^{3}$ ). Denote $V^{3}=f^{-1}\left(U^{3}\right)$. Then $\left.f\right|_{V^{3}}: V^{3} \rightarrow U^{3}$ is a proper degree one map of open 3 manifolds. By hypothesis, we conclude that $U^{3}$ embeds in $S^{3}$. It follows from Theorem 1 of [3] that $K$ can be engulfed and therefore $M^{3}$ is standard.

Only if: We will produce a compactification of $U^{3}$ which, modulo the P.C., is $S^{3}$. First the existence of $f$ implies the existence of virtual disks in $U^{3}$ with certain properties (which will not be stated here). The Lemma then implies the existence of embedded virtual disks with certain properties. Given an embedded virtual disk, $\check{D} \subset U^{3}$, we form a "precompactification" of $U^{3}$ (denoted $U_{D}^{3}$ ) in the following way: let $N(\breve{D})$ be a product neighborhood of $\check{D}$ and let $h: N(\check{D})$ $\rightarrow\left(D^{2}-X\right) \times I$ be the obvious homeomorphism (where $X$ is as in the definition of virtual disk). Let $U_{D}^{3}=U^{3} \cup_{h}\left(D^{2} \times I\right)$. $U_{D}^{3}$ is then an open, acyclic 3-manifold with $U^{3} \subset U_{D}^{3}$ and in which $\partial \check{D}$ bounds a disk.

Repeating this procedure infinitely many times (for the appropriate choice of virtual disks) we obtain an open 3-manifold, $W^{3}$, with the property that given any compact $X \subset W^{3}$ there exists a compact 1 -connected 3-submanifold $M^{3}$ with $X \subset M^{3} \subset W^{3}$. The P.C. implies $M^{3}$ is a punctured 3-sphere and it follows that the Freudenthal compactification of $W^{3}$ is $S^{3}$.

Note. Theorem 1 is still true if the word "irreducible" is deleted. However the "if" direction is then trivial.

Reformulation. Denote: $U=$ the class of all open, irreducible, acyclic 
3-manifolds, $V=$ the class of all open 3-manifolds embeddable in $S^{3}, W=$ the class of all open 3-manifolds each of which is the degree one proper image of some element of $V$.

We can then reformulate Theorem 1 as

THEOREM 1'. P.C. iff $u \cap w \subset U$.

In fact, in the statement of Theorem $1^{\prime}$ we can replace $U$ by any subclass $U_{0}$ of $U$ such that given $M^{3}$, a homotopy 3-sphere, there exists a Heegaard splitting, $K$, of $M^{3}$ and $U \in U_{0}$ such that $K \subset U \subset M^{3}$. There exist interesting examples of such a $U_{0}$ (see [4], [5] and [1]).

The compactification procedure used in the proof of Theorem 1, can be used to prove

THEOREM 2. Let $U^{3}$ be a noncompact 3-manifold. Assume (i) P.C., (ii) $U^{3}$ is acyclic at $\infty$ and (iii) there exists a 3-manifold $V^{3}$, embeddable in $S^{3}$, and a degree one, proper map $f:\left(V^{3}, \partial V^{3}\right) \rightarrow\left(U^{3}, \partial U^{3}\right)$. Then $U^{3}$ embeds in some compact 3-manifold.

Theorem 2 together with results of [1] (in particular Lemmas 1 and 2 and Theorem 1) yield

COROLlARY. P.C. only if any compact generalized 3-manifold without boundary, whose nonmanifold set is 0-dimensional, has a resolution.

We refer the reader to [1] (in which a weaker version of the Corollary was proven) for definitions. Details of the proofs will appear elsewhere.

\section{REFERENCES}

1. M. G. Brin and D. R. McMillan, Jr., Generalized three-manifolds with zero-dimensional non-manifold set, SUNY at Binghamton and University of Wisconsin at Madison (preprint).

2. E. M. Brown and C. D. Feustel, On proper essential embeddings of planes in 3manifolds, Notices Amer. Math. Soc. (1979) 766-G14 (preprint).

3. D. R. McMillan, Jr. and T. L. Thickstun, Open 3-manifolds and the Poincaré conjecture, Topology 19 (1980), 313--320.

4. V. Poenaru, A remark on simply connected 3-manifolds, Bull. Amer. Math. Soc. 80 (1974), 1203-1204.

5. T. L. Thickstun, Open contractible manifolds in homotopy 3-spheres, Inst. Hautes Études Sci. Publ. Math., 1977 (preprint).

SCHOOL OF MATHEMATICS AND COMPUTER SCIENCE, UNIVERSITY COLLEGE OF NORTH WALES, BANGOR, LL57 2 UW, UNITED KINGDOM 